Supporting Information

\title{
Helix Dipole and Membrane Electrostatics Delineate Conformational Transitions in the Self-assembly of Amyloidogenic Peptides
}

Qiuchen Zheng, ${ }^{\dagger}$ Senegal N. Carty ${ }^{\dagger}$ and Noel D. Lazo*

Carlson School of Chemistry and Biochemistry, Clark University, 950 Main Street, Worcester, Massachusetts 01610 , United States

†These authors contributed equally to this work.

*To whom correspondence should be addressed. 
A

$100 \%$ DOPC

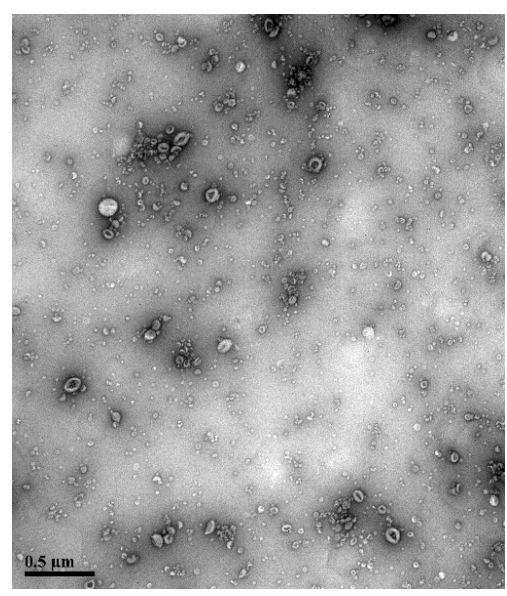

100\% DOPS

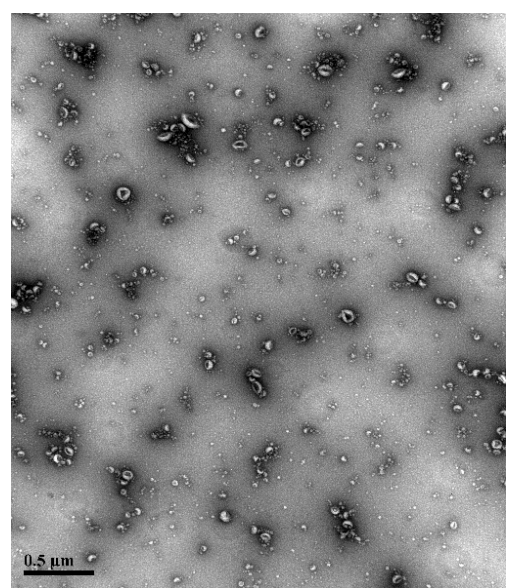

DOPC/DOPS

(7:3, mol:mol)

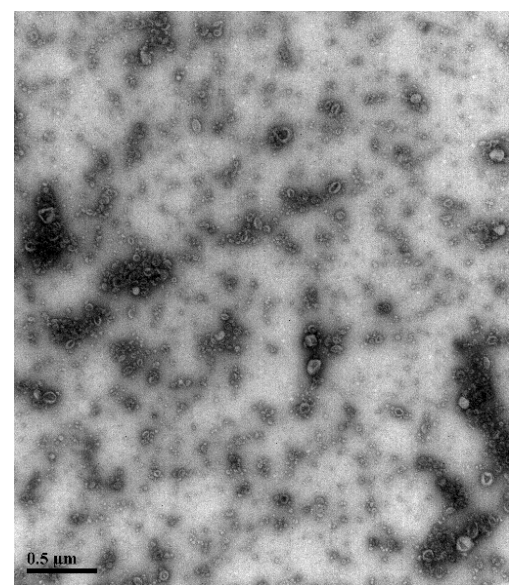

B

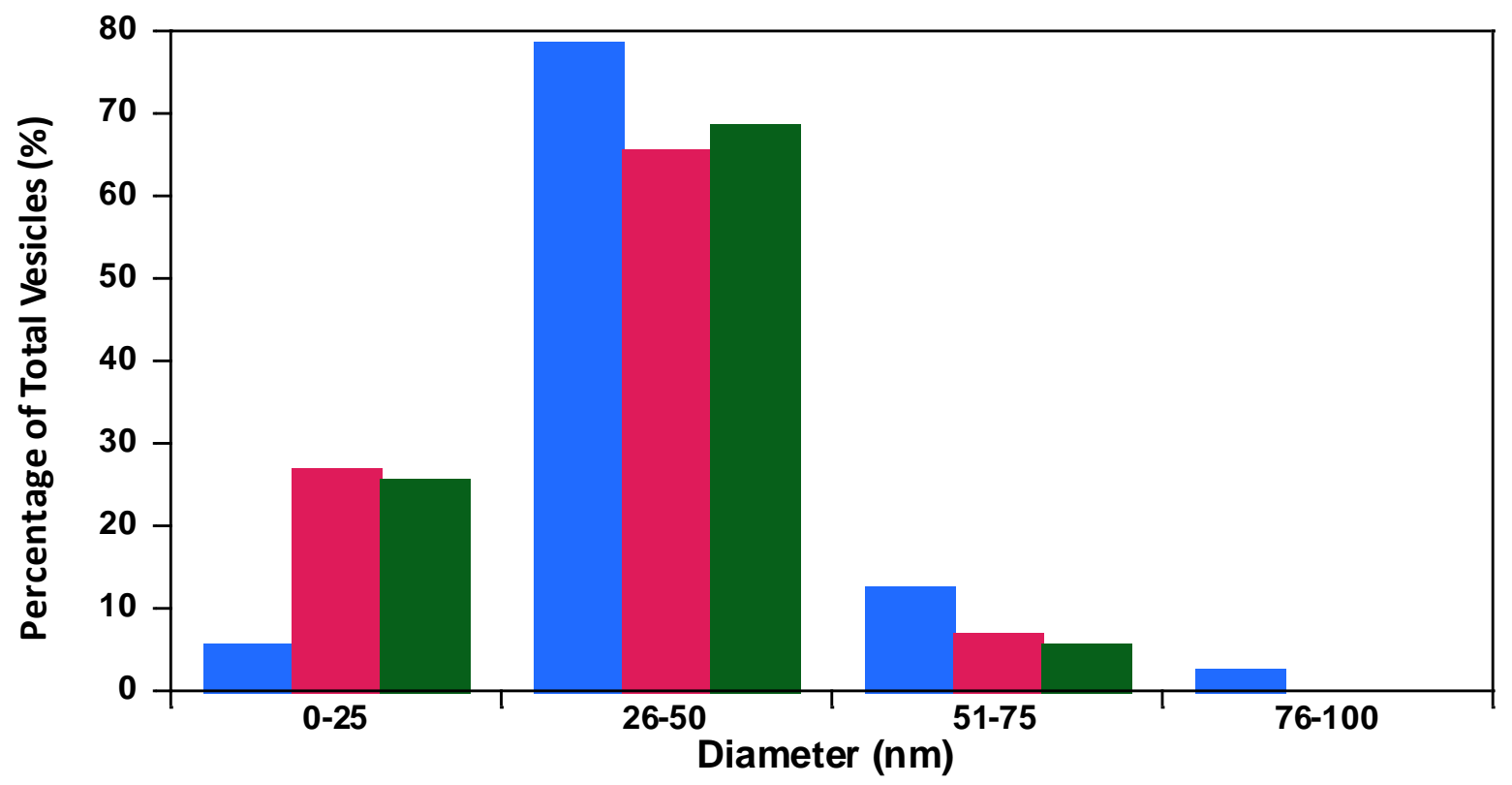

Figure S1. Characterization of SUVs only. (A) TEM images of the SUVs composed of 100\% DOPC (left), 100\% DOPS (middle), and DOPC/DOPS (7:3, mol:mol) (right). The scale bar corresponds to $0.5 \mu \mathrm{m}$. (B) Distribution of vesicle diameters in 100\% DOPC (blue), 100\% DOPS (red), and DOPC/DOPS (7:3, mol:mol) (green). 


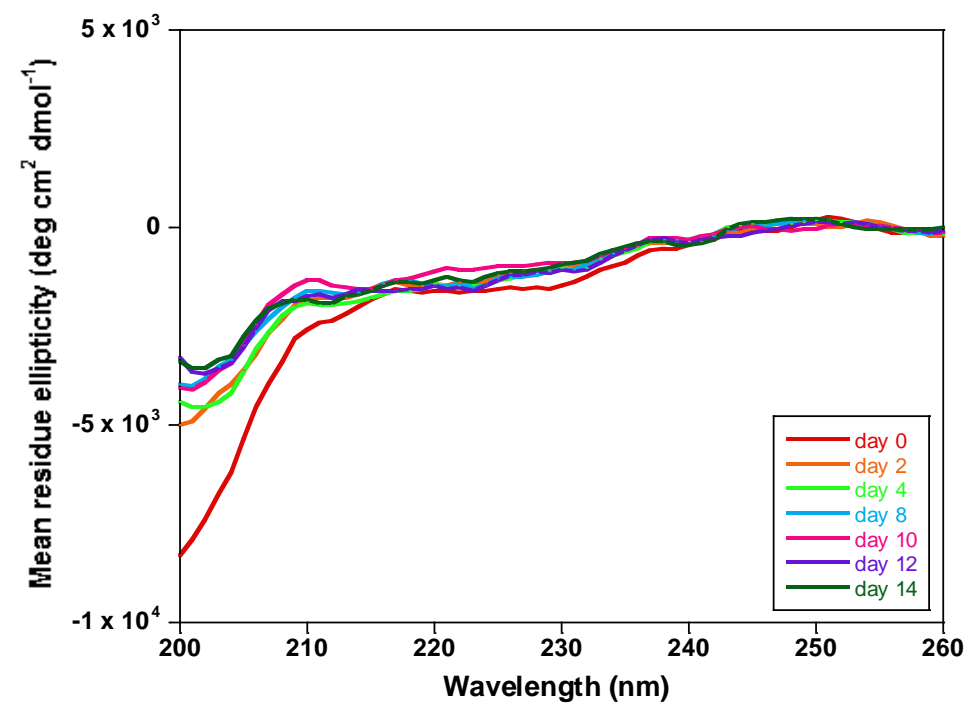

Figure S2. CD spectra of IAPP[11-25(S20G)-NH $\left.\mathrm{NH}_{2}\right]$ in pure buffer. The concentration of IAPP $\left[11-25(\mathrm{~S} 20 \mathrm{G})-\mathrm{NH}_{2}\right]$ in the sample was set at $30 \mu \mathrm{M}$. 

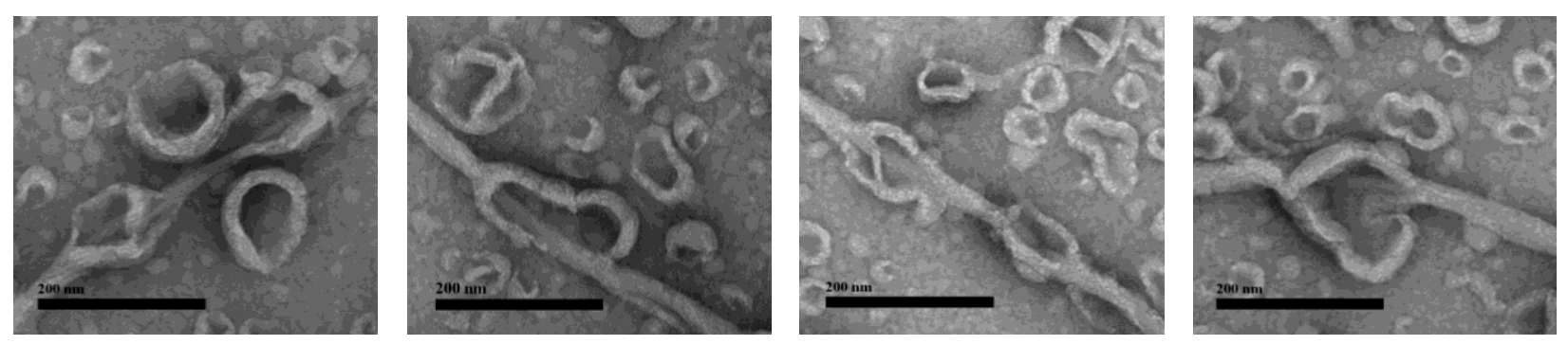

Figure S3. TEM characterization of circular dichroic samples of IAPP $\left.111-25(\mathrm{~S} 20 \mathrm{G})-\mathrm{NH}_{2}\right]$ in the presence of SUV composed of DOPC/DOPS (7:3, mol:mol) at higher magnification. The scale bar corresponds to $200 \mathrm{~nm}$. 

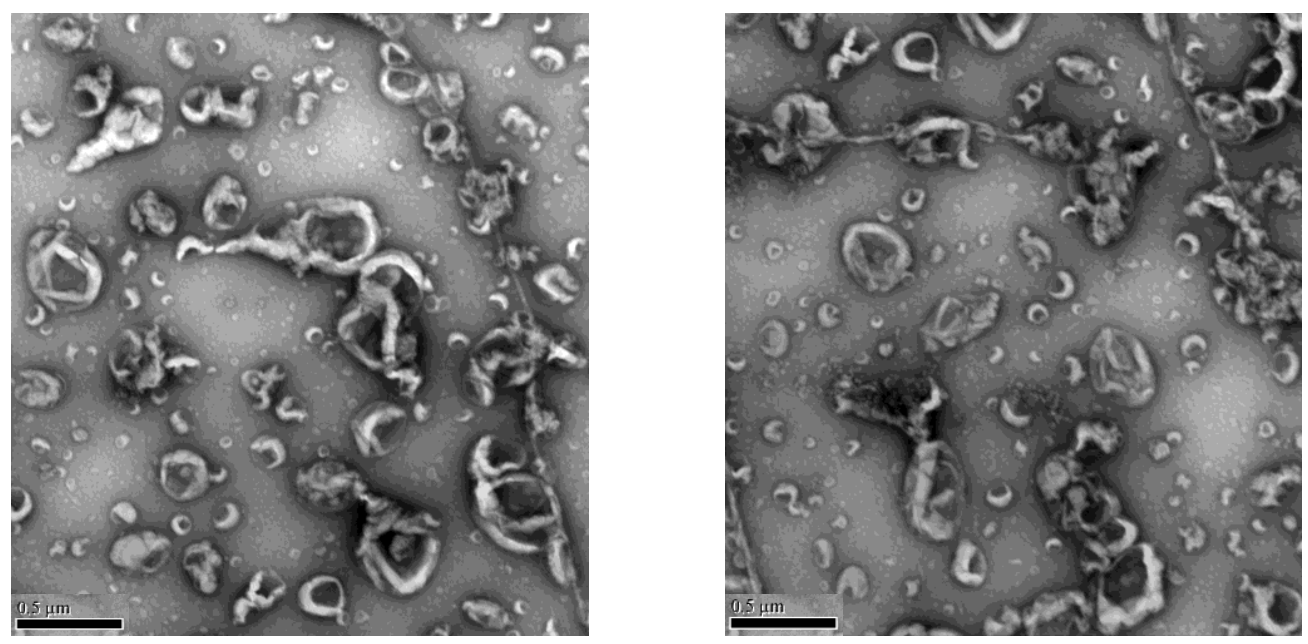

Figure S4. TEM characterization of circular dichroic samples of IAPP[11-25(S20G)-NH2] in the presence of SUV composed of $100 \%$ DOPS, presenting vesicles with roughened surfaces. Scale bar corresponds to $0.5 \mu \mathrm{m}$. 